\title{
Fog Computing-Based Differential Positioning Method for BDS
}

\author{
Lina Wang $\mathbb{D}^{1,2,3}$ and Linlin $\mathrm{Li}^{1}$ \\ ${ }^{1}$ School of Computer and Communication Engineering, University of Science and Technology Beijing, Beijing 100083, China \\ ${ }^{2}$ Beijing Engineering and Technology Research Center for Convergence Networks and Ubiquitous Services, \\ University of Science and Technology Beijing, Beijing 100083, China \\ ${ }^{3}$ Beijing Key Laboratory of Knowledge Engineering for Materials Science, Beijing, China \\ Correspondence should be addressed to Lina Wang; wln_ustb@126.com
}

Received 16 April 2018; Accepted 6 June 2018; Published 5 July 2018

Academic Editor: Ke Xiong

Copyright (C) 2018 Lina Wang and Linlin Li. This is an open access article distributed under the Creative Commons Attribution License, which permits unrestricted use, distribution, and reproduction in any medium, provided the original work is properly cited.

\begin{abstract}
As one of the four global satellite navigation and positioning systems, BeiDou satellite navigation system (BDS) has received increasingly more attention. The differential positioning technology of BDS has greatly enhanced its accuracy and meets the needs of high-precision applications, but its positioning time still has much room for improvement. Fog computing allows the use of its services with low latency and mobility support to make up for the disadvantages of differential positioning algorithm. The paper proposes the fog computing-based differential positioning (FCDP) method which introduces fog computing technology to BDS. Compared with the original data center-based differential positioning (DCDP) method, the simulation results demonstrate that the FCDP method decreases the latency of positioning, while assuring the positioning accuracy.
\end{abstract}

\section{Introduction}

BeiDou satellite navigation system (BDS) is a self-developed and independently operated global satellite navigation and positioning system in China [1-4]. In recent years, the industrialization of BeiDou civil application and its promotion work have achieved remarkable results with far-reaching effects [4]. Since 2012, when the system officially serves the Asia Pacific region, the BDS has been applied in various fields, including transportation, marine fisheries, hydrological monitoring, and weather forecasting [5]. As BDS plays an important role in many fields, it is very important to provide fast and accurate positioning algorithm to improve the system competitiveness and promote the development of the system.

Due to eliminating some common errors in the system, the differential positioning technology has been commonly used in positioning tasks and applications with higher accuracy requirements [6-8]. With this technology, all the differential data of base stations are sent to the data center and then return to mobile station after calculation. Due to the existence of the data center, the differential positioning method provides multilevel authentication for users and improves the computing ability $[9,10]$. Despite the fact that the data center-based differential positioning (DCDP) method provides many benefits, there are still some challenges such as increases in delay, the waste of bandwidth, and privacy concerns, which is not good for quick positioning and the development of BDS [11]. In addition, with the development of the BDS, the construction of the base station will surely increase construction efforts to keep pace with the needs of the business, and the scalability of the DCDP system will be limited by the performance of the data center. Once the data center is attacked, the overall system performance will be greatly reduced.

To reduce the positioning time and solve all of the above problems caused by the data center, many scholars have conducted studies on algorithms or methods [12-15]. The paper [12] proposed an algorithm based on congestion control, i.e., Random Early Detection (RED) gateways, which could avoid unnecessary queue delay, while the general reduction in delay does not offer the best results. The paper [13] proposed the cutting payload (CP) mechanism to process timeouts but the CP mechanism is limited to hardware conditions. The 
paper [14] examined the effectiveness of alternative TCP and Ethernet-level strategies in mitigating the TCP throughput collapse but at the cost of seriously increasing the input costs. The paper [15] proposed a global data transfer scheduler at the application layer but it will destroy synchronization.

The above approaches are for congestion, queuing, and other issues caused by the data center in order to provide improvement. However, the centralized mode is still adopted and does not fundamentally solve the potential problem caused by bidirectional transmission, especially the transmission delay caused by the uplink and downlink. In other words, due to the constraint of triangle inequalities, the link delay through the intermediate node forwarding is greater than the direct link delay between two points. To resolve the disadvantages of the data center, fog computing has recently emerged. Fog computing is considered as a cloud server operating at the edge of the network, offering special services that require network context information, location awareness, and ultra-low latency [16].

Since the features of fog computing could satisfy the demands of BDS exactly, the paper integrates fog computing to BDS for quicker and even real-time positioning processing and proposes a fog computing-based differential positioning (FCDP) method to offload computing tasks and decrease delay. Meanwhile, the original high-precision feature of the DCDP is not affected.

The reminder of this paper is organized as follows. Section 2 introduces the current differential positioning model and DCDP method of BDS. Section 3 describes the FCDP proposed by this paper and formulates the calculation progress. Section 4 presents the experimental results and analyses. Finally, Section 5 concludes the paper.

\section{The Carrier Phase Differential Positioning Principle with the Data Center}

According to the information used, differential positioning algorithm can be divided into four types: position differential positioning, pseudo-range differential positioning, phase smooth pseudo-range differential positioning, and carrier phase differential positioning [17]. Among them, carrier phase measurements of carrier phase differential positioning allow the measurement of short baselines with an inaccuracy as low as a few centimeters [18]. Therefore, the paper adopts the carrier phase difference as an example to introduce the differential positioning principle.

There are two types of receivers: base station receiver and mobile receiver. The base station is used as a benchmark to correct the common error between mobile receiver and base receiver. The mobile receiver is usually used as a target to locate devices such as mobile phone or the user's other device.

The base station and mobile receiver simultaneously observe the ephemeris information of BeiDou satellites. The observation equations of satellite $j$ are as follows [19]:

$$
\begin{aligned}
& \rho_{b}^{j}=\lambda\left(N_{b}^{j}+\varphi_{b}^{j}\right)-c\left(d \tau_{b}-d \tau^{j}\right)-d \rho_{b}^{j}+d I_{b}^{j}-d T_{b}^{j} \\
& \rho_{u}^{j}=\lambda\left(N_{u}^{j}+\varphi_{u}^{j}\right)-c\left(d \tau_{u}-d \tau^{j}\right)-d \rho_{u}^{j}+d I_{u}^{j}-d T_{u}^{j}
\end{aligned}
$$

Here, $\rho_{*}^{j}$ represents the real distance between the satellite $j$ and the receiver; $\lambda$ is the wavelength of carrier; $N_{*}^{j}$ denotes the number of circumference between receiver and satellite $j$; $\varphi_{*}^{j}$ is the period that is less than one circumference; $c$ is the speed of the electromagnetic wave; $d \tau_{*}$ is the clock deviation of the receiver; $d \tau^{j}$ is the deviation of satellite $j$; $d \rho_{*}^{j}$ is the error caused by satellite calendar; $d I_{*}^{j}$ is ionosphere error; and $d T_{*}^{j}$ is tropospheric error.

When the distance between base station and the mobile station is of short or medium baseline (less than $100 \mathrm{~km}$ ), $d \rho_{b}^{j} \approx d \rho_{u}^{j}, d I_{b}^{j} \approx d I_{u}^{j}, d T_{b}^{j} \approx d T_{u}^{j}[20]$. Therefore,

$$
\rho_{u}^{j}-\rho_{b}^{j}=\lambda\left(\varphi_{u}^{j}-\varphi_{b}^{j}\right)-c\left(d \tau_{u}-d \tau_{b}\right)+\lambda\left(N_{u}^{j}-N_{b}^{j}\right)
$$

$\Delta(\bullet)$ is ordered to replace the single difference between the base station and the mobile station, and then

$$
\Delta \rho_{u b}^{j}=\lambda \Delta \varphi_{u b}^{j}-c \Delta d \tau_{u b}+\lambda \Delta N_{u b}^{j}
$$

For the convenience of programming calculations, (3) is linearized [21].

$$
H_{u}^{j} \Delta p_{u}+\hat{\rho}_{u}^{j}-\rho_{b}^{j}=\lambda \Delta \varphi_{u b}^{j}-c \Delta d \tau_{u b}+\lambda \Delta N_{u b}^{j}
$$

where $H_{u}^{j}$ denotes the direction vector for the mobile station and satellite $j ; \Delta p_{u}$ is the coordinate correction of the mobile receiver; and $\hat{\rho}_{u}^{j}$ represents the approximate distance between satellite $j$ and mobile station.

The above formula is the single-difference model of BDS, while the positioning error is still influenced by clock deviation that can be eliminated through calculating the quadratic difference between different satellites [22].

For satellite $k$, the single-difference equation is [21]

$$
H_{u}^{k} \Delta p_{u}+\hat{\rho}_{u}^{k}-\rho_{b}^{k}=\lambda \Delta \varphi_{u b}^{k}-c \Delta d \tau_{u b}+\lambda \Delta N_{u b}^{k}
$$

The second difference between (4) and (5) is

$$
\begin{aligned}
& \left(H_{u}^{j}-H_{u}^{k}\right) \Delta p_{u}+\left(\hat{\rho}_{u}^{j}-\hat{\rho}_{u}^{k}\right)-\left(\rho_{b}^{j}-\rho_{b}^{k}\right) \\
& =\lambda\left(\Delta \varphi_{u b}^{j}-\Delta \varphi_{u b}^{k}\right)+\lambda\left(\Delta N_{u b}^{j}-\Delta N_{u b}^{k}\right)
\end{aligned}
$$

$\nabla \Delta(\bullet)$ is ordered as a double difference of different satellites, and (6) can be simplified as (7)

$$
\begin{gathered}
\left(H_{u}^{j}-H_{u}^{k}\right) \Delta p_{u}+\left(\hat{\rho}_{u}^{j}-\hat{\rho}_{u}^{k}\right)-\left(\rho_{b}^{j}-\rho_{b}^{k}\right) \\
=\lambda \nabla \Delta \varphi_{u b}^{j k}+\lambda \nabla \Delta N_{u b}^{j k}
\end{gathered}
$$

where $\nabla \Delta \varphi_{u b}^{j k}$ is phase observation error and $\nabla \Delta N_{u b}^{j k}$ is double-difference integer ambiguity.

Let $L_{u b}^{j k}=\lambda \nabla \Delta \varphi_{u b}^{j k}+\left(\rho_{b}^{j}-\rho_{b}^{k}\right)-\left(\hat{\rho}_{u}^{j}-\hat{\rho}_{u}^{k}\right)$; the error equation form of (7) can be simplified as

$$
\nabla \Delta v_{u b}^{j k}=\left(H_{u}^{j}-H_{u}^{k}\right) \Delta p_{u}-\lambda \nabla \Delta N_{u b}^{j k}-L_{u b}^{j k}
$$

Here, $L_{u b}^{j k}$ is the correction value to revise the common error. 


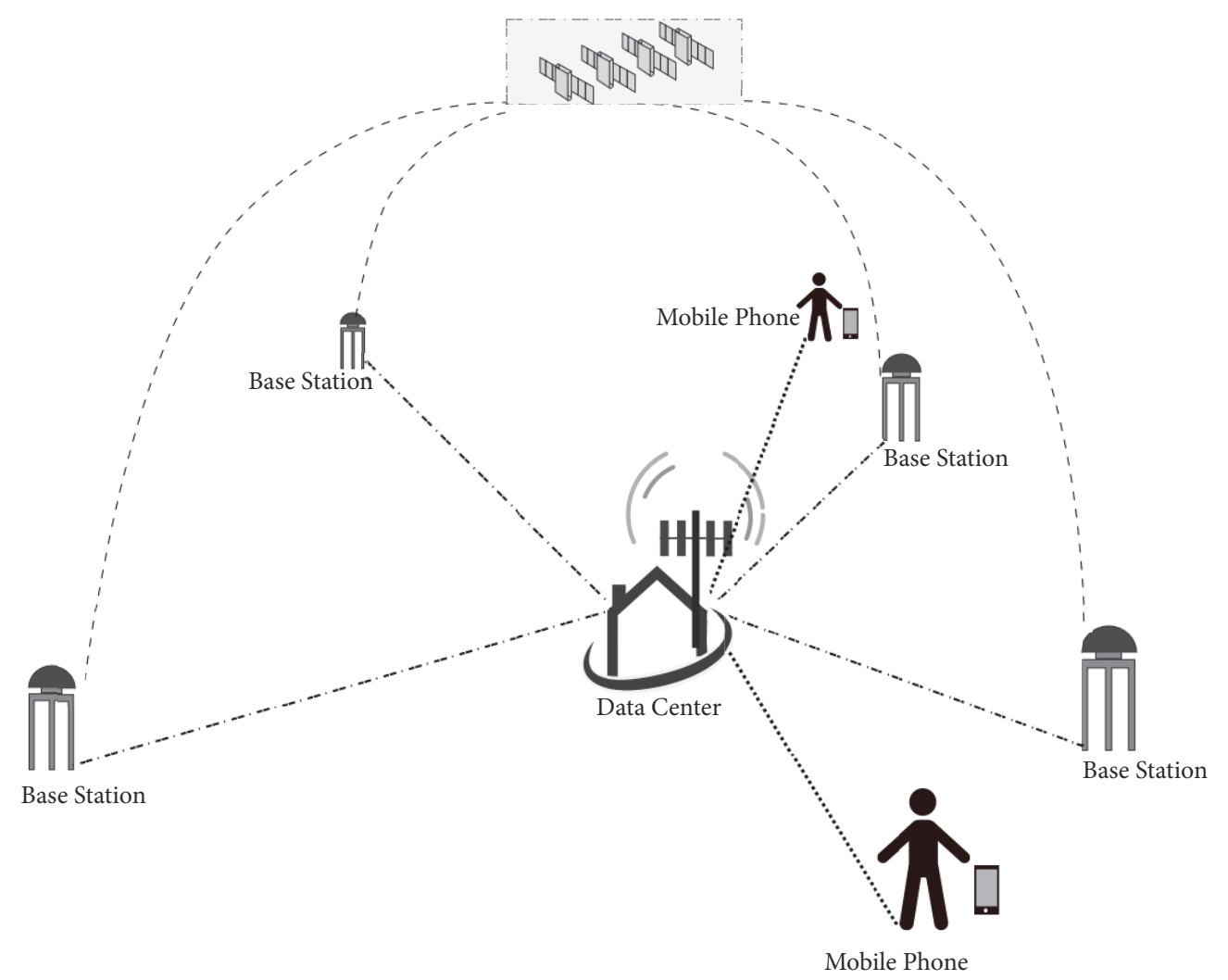

Figure 1: DCDP method model.

Within the positioning area, multiple base stations constitute the continuously operating reference stations (CORS) system [23]. All of the correction value of different base stations should be sent to data center to calculate the more suitable final value that corrects the mobile station, called the DCDP method. The data center calculates the final differential correction synthetically based on several principles such as weighted average method, partial derivative method, and minimum variance method. Then, the data center sends the final value, which could revise the common error to the mobile receiver. The model of the DCDP system is shown in Figure 1.

As is shown in Figure 1, there is no direct connection between the base station and mobile station, but the exchanged data and calculated final information by the data center causes transmission delay, data congestion, packet loss, and other issues. The paper solves these issues by introducing fog computing.

\section{Fog Computing-Based Differential Positioning Method}

3.1. Fog Computing. According to the definition of Cisco, the level of fog calculation is at the edge of the network, and the equipment used can be a router or base station [24]. These devices do not have many resources such as CPU, memory, hard disk, or bandwidth as they do in the data center, but they are closer to the user. The response delay is lower when a user requests the service, and some data communications are already set between the user and the data information. In other words, fog computing is a highly virtualized middle layer between end users and the cloud. Similar to cloud computing, fog computing provides user data, computing, storage, and network services [25]. It is not centralized but close to the end devices. By lowering data resources from users to the edge of the network, users do not need to obtain resources from a distant cloud center, which can effectively reduce the traffic and delay when obtaining services, finally effectively reducing the network burden.

The fog computing system consists of multiple mutually independent fog computing nodes and can independently provide localization services for mobile terminals. There are three layers including terminal layer, fog layer, and cloud layer [26]. The service model of fog computing is shown in Figure 2.

The cloud layer and the fog layer provide services for end users. The cloud layer generally serves network users, while the fog layer mainly serves intelligent mobile terminal users. The terminal layer buffers the resources from the fog layer node in advance according to a certain strategy. Then the fog layer computed node has a certain storage space and computing capacity to handle the task request of the terminal layer. Furthermore, the fog layer node can be automatically located and then, the nearest neighboring fog server is found, so that the terminal's data request can always obtain a response from the nearest fog server [26].

The fog computing architecture has three parts that match the service model including fog computing edge storage 


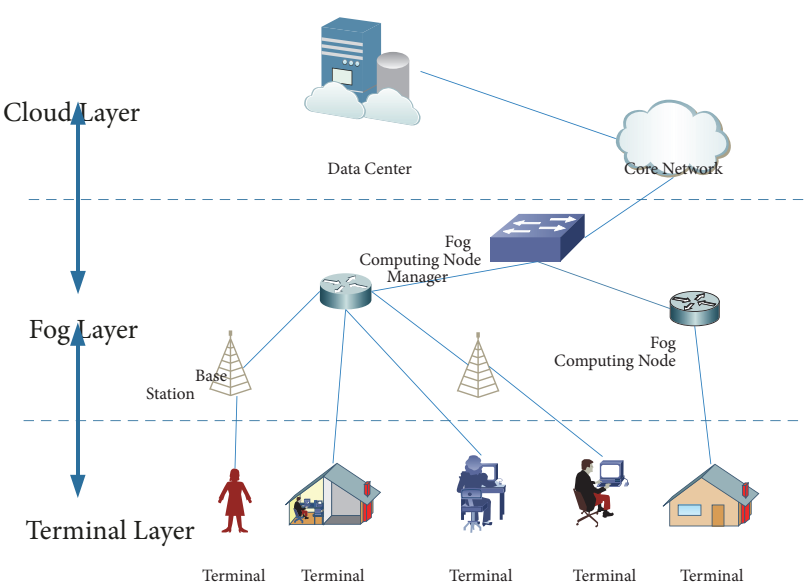

Figure 2: Service model of fog computing.

nodes (fog computing nodes), fog computing node manager, and fog computing network. The three parts correspond to three layers [26].

(1) Fog Computing Edge Storage Nodes. The fog computing edge storage node or server is a miniaturized hardware device that is disposed locally and provides localized services for mobile terminal users through wireless access. The device integrates high-density wireless network access technology, large data and low-delay channel transmission technology, and localized storage and computing technologies for cloud computing front-end hardware devices. The fog computing edge storage node is located at the edge of the network and is the closest component to the end user's fog computing system. Its data content comes from the cloud platform's data center, and it is updated intelligently according to the user's needs. The end user needs only one hop to be able to obtain the storage data service in the cloud-computing node. The fog computing nodes can be deployed on mobile vehicles, such as intercity buses, tourism operators, trains, and ships, to provide localization (without connecting to cloud data centers), high-speed, and low-cost services for users.

(2) Fog Computing Node Manager. The corresponding management program of the fog computing node is called the fog computing manager. For example, the data object storage table is used to record the data objects, access time, and access data logs that are stored in the fog computing node. Every time the mobile terminal requests the service of edge node, the manager first looks up whether there is a record stored in the table. If so, the data object of the local fog computing node is returned to the terminal user directly and then the related content of the data storage table is updated; if not, within the specified time, the data object is requested to other nodes at a minimum cost. In addition, if the other fog computing nodes have the data object in the cache, the data will be provided to the user; and if not, the request will be forwarded to the cloud to look for the data and return to the user. Correspondingly, the fog computing manager will also make the appropriate record updates and decides whether to store the data object.

(3) Fog Computing Network. Fog computing network consists of geographically dispersed nodes and storage nodes. These nodes can communicate with each other through the fog network and perform data transmission according to a set routing algorithm, data distribution strategy, etc.

If a fog computing node finds the required data stored in other fog computing nodes, the fog computing node can obtain the data needed by fog computing network. At the same time, the fog computing node can also connect to the cloud or the source server through the Internet to obtain data objects. Furthermore, the cloud can timely monitor the content information change and then update the record correspondingly.

Due to wide distribution and high density, fog computing can quickly respond to end-user requests, handle tasks in real time, and shorten access delays. Furthermore, the fog nodes are at the edge of the network and close to the end user, so the user needs only one hop to obtain the various data resources needed. Because fog computing nodes do not have as much storage and computing space as cloud computing, certain strategies must be adopted to filter the fog computing nodes that can provide maximum value for terminals in the service area, to maximize the quality of the service to users.

3.2. FCDP Method. Due to the problems of DCDP method caused by the data center such as high latency, congestion, and packet loss, this paper proposes to apply fog computing to BDS. In the FCDP method, the data center was removed, and the base station is set as the fog layer to calculate the correction information. Then all the base stations within the positioning area broadcast the correction data incessantly. The mobile receiver in the area receives the correction information from the base stations and performs a comprehensive calculation. The model of FCDP is shown in Figure 3.

In the positioning area, all base stations can participate in positioning. However, if there are too many base stations, the factors that affect the accuracy of mobile receivers will also increase. In consideration of comprehensive elimination of mobile station errors and the stability of the reference station network, four reference stations are selected for positioning in this paper. Therefore, a suitable base station selection strategy is needed to minimize network losses and balance the delay between stations.

The base station selection algorithm of this paper considers three main indicators: transmission delay (distance between base station and mobile station), balance of base station load, and performance of the base station itself. It can be seen from (1) that the pseudo-range value is related to tropospheric and ionospheric effects, which have a strong correlation with distance [27]. Although the atmospheric noise of the base station and mobile receiver can be equal by default in the case of medium or short baseline, shorter baselines have higher similarity [27]. Therefore, the weight of 


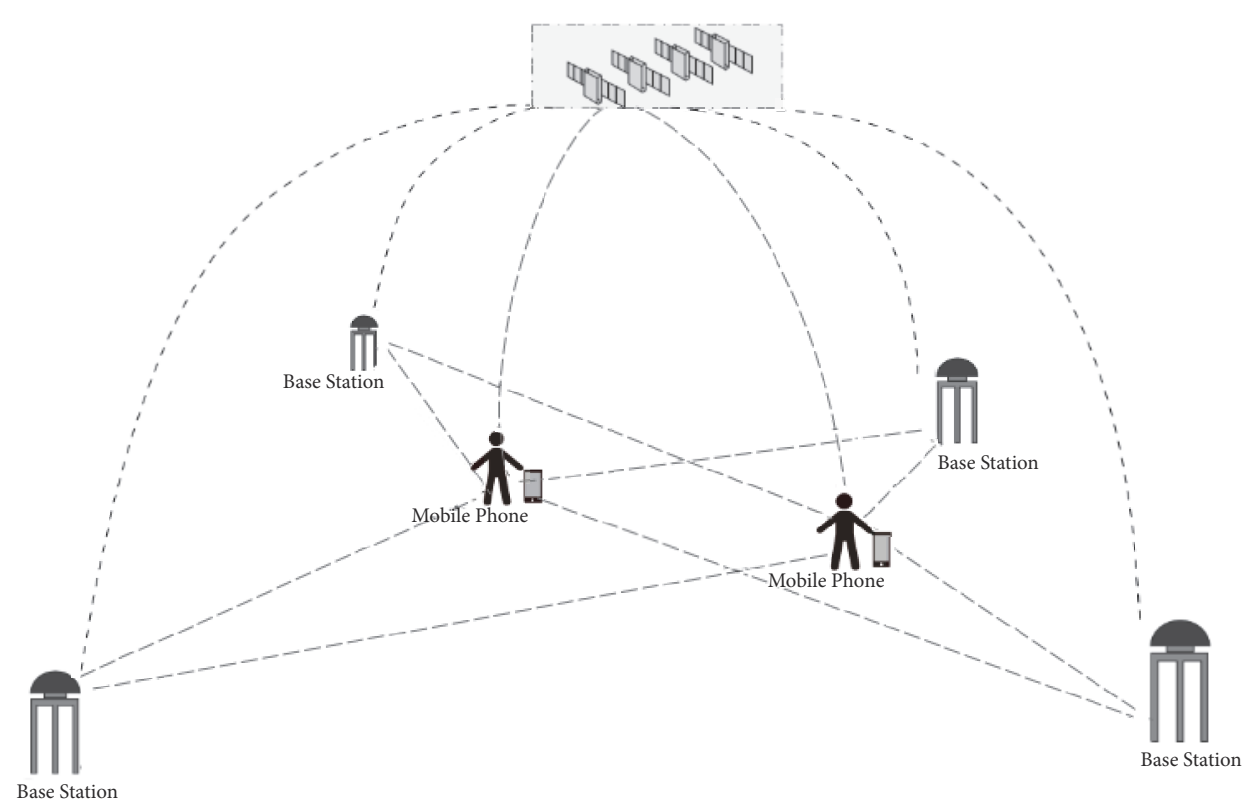

Figure 3: FCDP method model.

the base station closer to the mobile receiver should be higher. In addition, unbalanced node assignments will cause some base stations to be overloaded and increase the likelihood of node crashes. Finally, the performance of the base station itself also affects the trustworthiness of the parameters.

According to the above requirements, we need to find an optimal objective function to implement the selection strategy of the base station. The above purpose can be expressed as formula (9).

$$
J(u, i)=\text { penalty }(t(u, i)) * t(u, i) * a(q, i)
$$

where

$$
\text { penalty }(t(u, i))= \begin{cases}1, & t(u, i)<\varepsilon \\ \infty, & t(u, i) \geq \varepsilon\end{cases}
$$

$J(u, i)$ is the performance function of base station $i$ to mobile receiver $u ; t(u, i)$ is the time of transmitting $L_{u b_{i}}^{j k}$ to mobile receiver $u ; q$ is the selected rate of base station $i$, and $a(q, i)$ is the reliability of base station $i$ which is positively related to the selected rate. penalty $(t(u, i))$ is the penalty function for transmission time. When the transmission time exceeds the set valuee, the penalty function value will be infinite. Then, this base station will not be selected.

Based on the size of the performance function value, the top four base stations that are not infinitely involved are selected for positioning. Therefore, if the penalty function value is infinite, this base station will not be selected.
When observing satellite $j$ and $k$, the correction value of base station $i$ is ordered to (11).

$$
L_{u b_{i}}^{j k}=\lambda \nabla \Delta \varphi_{u b_{i}}^{j k}+\left(\rho_{b_{i}}^{j}-\rho_{b_{i}}^{k}\right)-\left(\hat{\rho}_{u}^{j}-\hat{\rho}_{u}^{k}\right)
$$

Then the final correction value is

$$
L_{f}^{j k}=\sum_{1}^{n} \frac{J(u, i)}{\sum_{1}^{n} J(u, i)} L_{u b_{i}}^{j k}
$$

where $n$ is the number of base stations participating in BDS positioning.

The weight of the correction number is inversely proportional to the distance between the base station and the mobile receiver, so the closer the distance is, the higher the credibility of the base station will be. Furthermore, the FCDP method has good antirisk ability. If the nearest base station fails to transmit information, or if any base station in the network fails, the remaining base stations can still form a network and calculate the final value based on the performance weighted.

After calculating the correction value, the error equation is

$$
\nabla \Delta v_{u b}^{j k}=\left(H_{u}^{j}-H_{u}^{k}\right) \Delta p_{u}-\lambda \nabla \Delta N_{u b}^{j k}-L_{f}^{j k}
$$

The matrix form of (13) is

$$
V=A X-L
$$




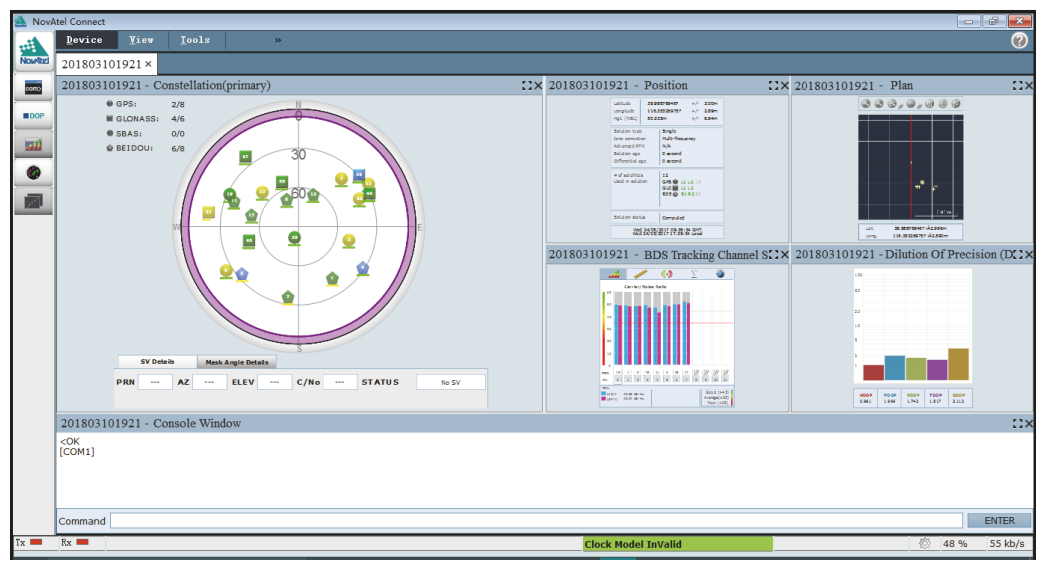

Figure 4: Interface of BeiDou satellites states.

Let $m$ be the number of observation satellites; then

$$
\begin{aligned}
A & =\left[\begin{array}{ccccc}
H_{u}^{2}-H_{u}^{1} & -\lambda & 0 & \cdots & 0 \\
H_{u}^{3}-H_{u}^{1} & 0 & -\lambda & \cdots & 0 \\
\vdots & \vdots & \vdots & \ddots & \vdots \\
H_{u}^{n}-H_{u}^{1} & 0 & 0 & \cdots & -\lambda
\end{array}\right], \\
X & =\left[\begin{array}{c}
\Delta p_{u} \\
\nabla \Delta N_{u b}^{j k}
\end{array}\right] \\
L & =\left[L_{f}^{12}, L_{f}^{13}, \cdots, L_{f}^{1 m}\right]^{T}
\end{aligned}
$$

Then, when the number of observation satellites is greater than or equal to 4 , the final receiver position can be calculated using the weighted least-squares algorithm. To weaken the influence of the correlation caused by the reference satellites, the chosen weight matrix usually uses the covariance inverse $Q^{-1}$ of the carrier observations.

Setting $R=Q^{-1}$, then the solution value of weighted leastsquares (WLS) algorithm is (16) [28].

$$
X=\left(A^{T} R A\right)^{-1} A^{T} R L
$$

Combined with the advantages of fog calculation, the FCDP method that removes the data center decreases the positioning delay.

\section{Numerical Results and Analyses}

To ensure the high reliability of the simulation experiment, this paper collected the satellite ephemeris information in the real environment as original positioning data through OEM6 ${ }^{\circledR}$ Family Firmware which produced by NovAtel company, a subsidiary company affiliated with Sweden Hexagon Company. The satellite status information can be obtained using the host computer software. As is shown in Figure 4 , the current satellite conditions information is shown intuitively by data collection. The acquired satellite ephemeris information converted into RINEX format can be used for positioning solution. Through the ephemeris parameters, we can obtain the coordinates of visible satellites used for positioning calculation.

4.1. Positioning Accuracy Comparison. When the coordinates of satellites in the CGCS2000 coordinate system are obtained, the positioning error of the DCDP and FCDP methods can be calculated using the weighted least square algorithm. As is shown in Figure 5, the absolute error of three axes and the root mean square error (RMSE) of these two methods are similar and all are below to $1 \mathrm{~m}$, while the RMSE of FCDP is more stable. The results demonstrate that, due to the selection strategy of the base stations, the positioning accuracy is more stable and have no big ups and downs.

Figure 6 shows the PDF of the DCDP and FCDP methods. As we can see, the FCDP method has more concentrated distribution, while the DCDP method has a slightly longer tail. It means that the FCDP method has better stability, rarely undulating largely in the same long-term duration.

4.2. Delay. In the FCDP method, there is a single-hop communication delay between the user and base stations, whereas the DCDP system has a two-hop communication delay that includes transmitting data to the server and sending the final result to the mobile receiver. The known singular delay of transmitting data is $270 \mathrm{~ms}$. Thus, the delay of the FCDP method with transmitting data is approximately $270 \mathrm{~ms}$, while the delay of the DCDP method is approximately $540 \mathrm{~ms}$. Therefore, the FCDP method has a shorter response time during transmission; theoretically, the delay is $270 \mathrm{~ms}$ shorter than that of the DCDP method.

The delay of the DCDP and FCDP methods is shown in Figure 7. Figure 7 illustrates that the delay of the FCDP method is less than $490 \mathrm{~ms}$ approximately and $260 \mathrm{~ms}$ shorter than the DCDP method. It means that the FCDP method can achieve faster positioning result, which has great significance especially in real-time positioning and mobile navigation fields. 

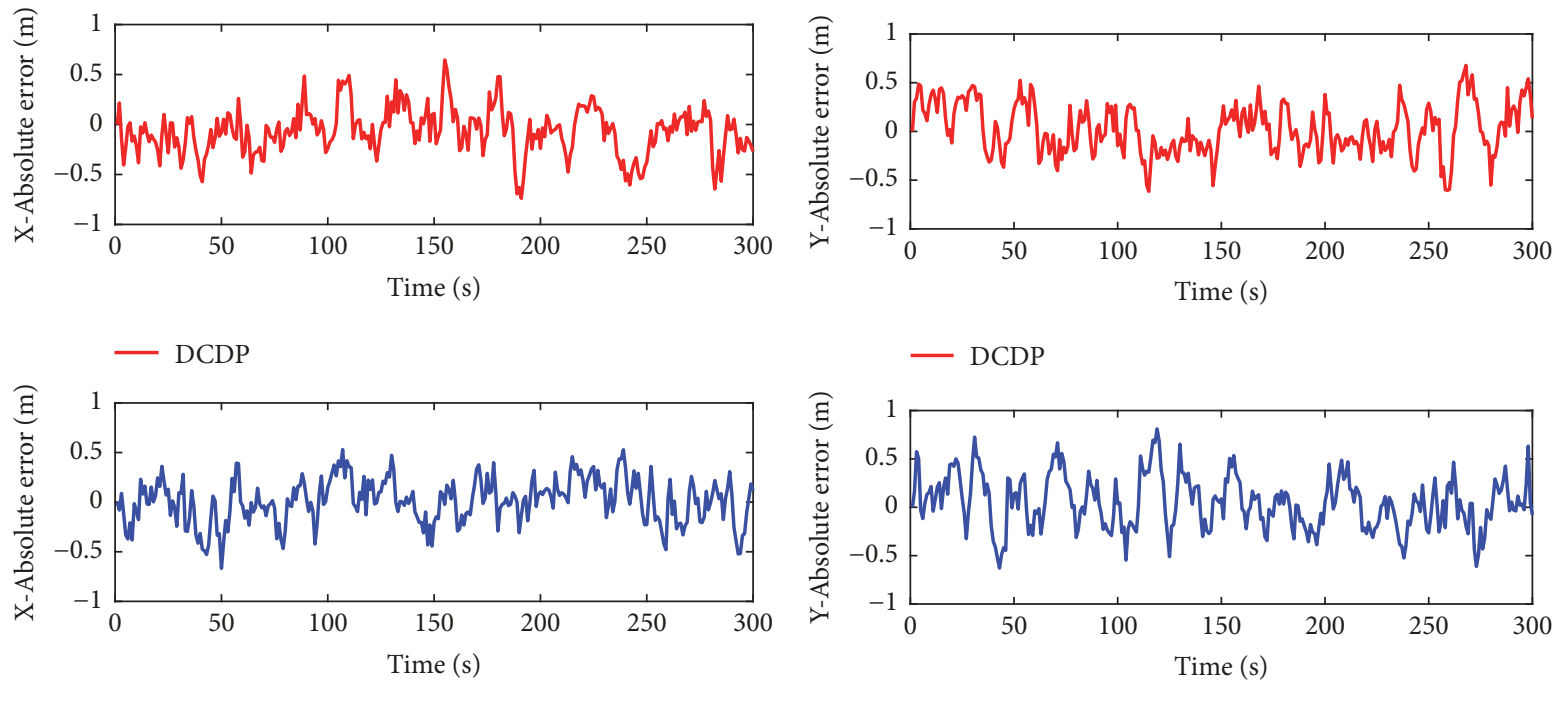

- FCDP

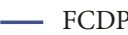

(a)
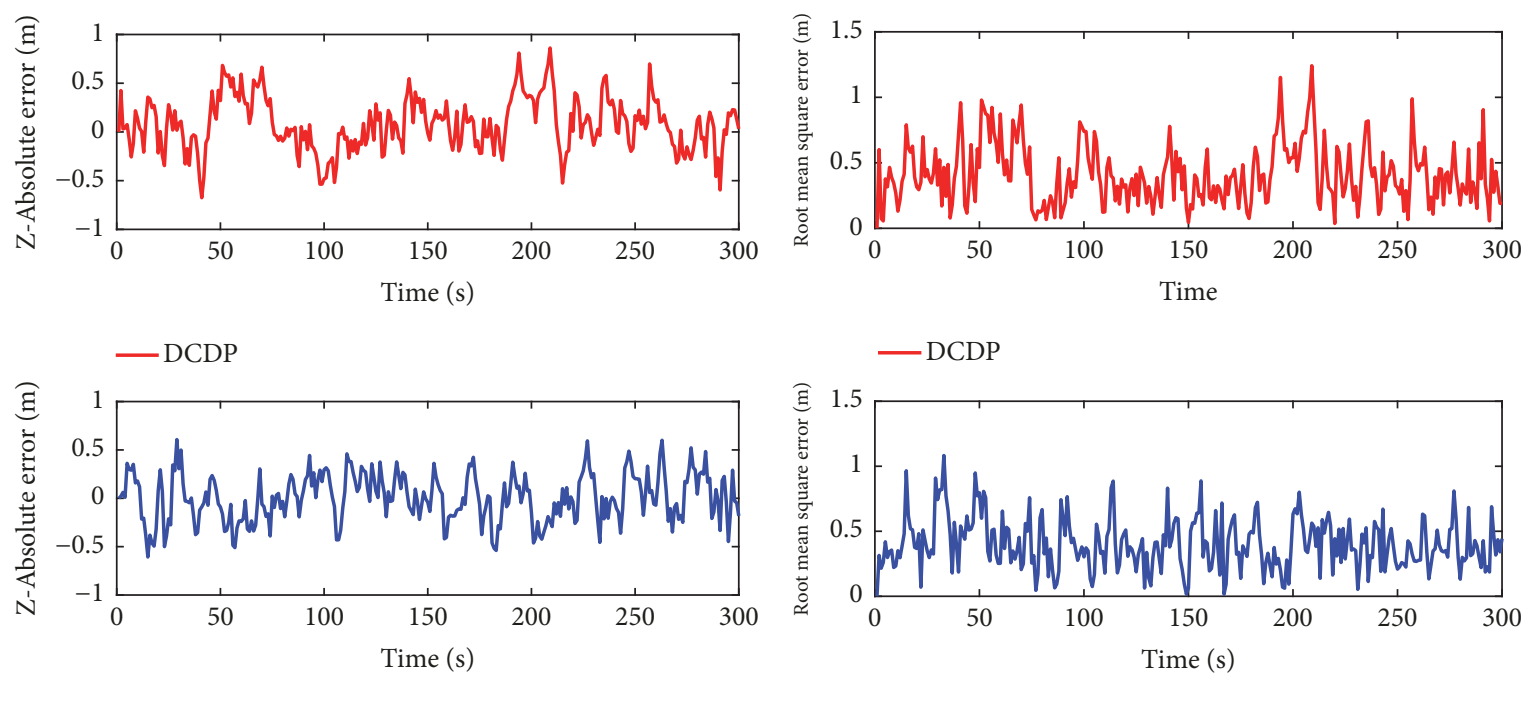

- FCDP

(c)

(d)

FIGURE 5: Error of DCDP and FCDP methods.

Consequently, the FCDP method is better than the DCDP method in terms of delay, has more stable accuracy range, and is beneficial for fast and accurate positioning of BDS. Furthermore, due to multiple fog computing nodes participating in the calculation, the data pressure is greatly shared and avoids server crashes, which are efficiently constructive to solve a large number of positioning requests and responses.

\section{Conclusions}

In recent years, new technology and application requirements have emerged continuously. Researchers are required to constantly learn and promote these applications from a new perspective in order to maximize the potential of BDS. The emergence of fog computing technology in the form of "decentralization" can be a way to solve the delay, congestion, and packet loss caused by the "center" of BDS. The FCDP method proposed in this paper combines the emerging fog computing technology. Under the condition of guaranteeing the accuracy of the original algorithm, the positioning delay is reduced, which is good for quick positioning. In addition, the networking of the fog computing layer also enhances the stability of the algorithm. Even if some base stations fail to transmit data effectively, the remaining base stations can still form a network without affecting the final data calculation. The simulation results demonstrate that the FCDP method has low latency and guaranteed accuracy which provide insights for improving response times. 


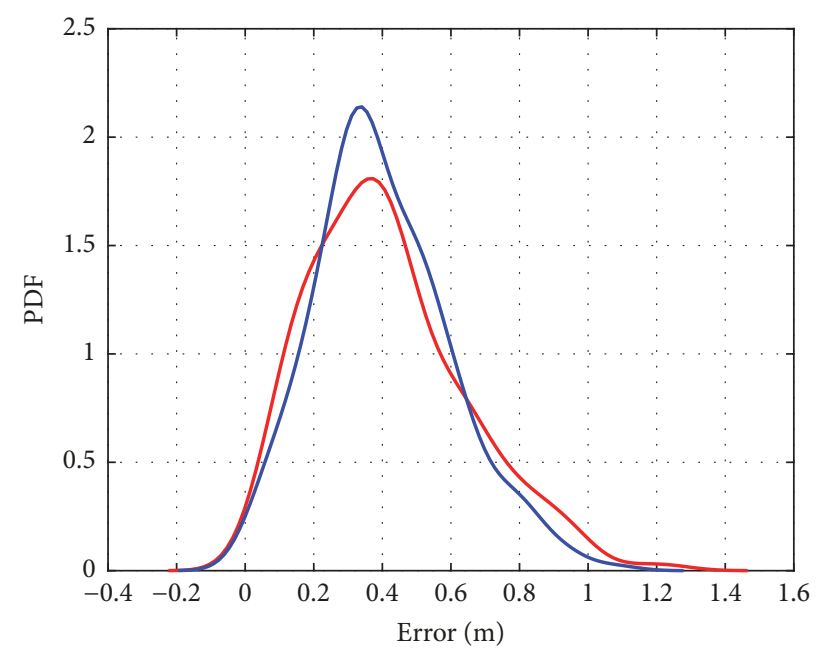

— DCDP
- FCDP

FIGURE 6: PDF of DCDP and FCDP methods.
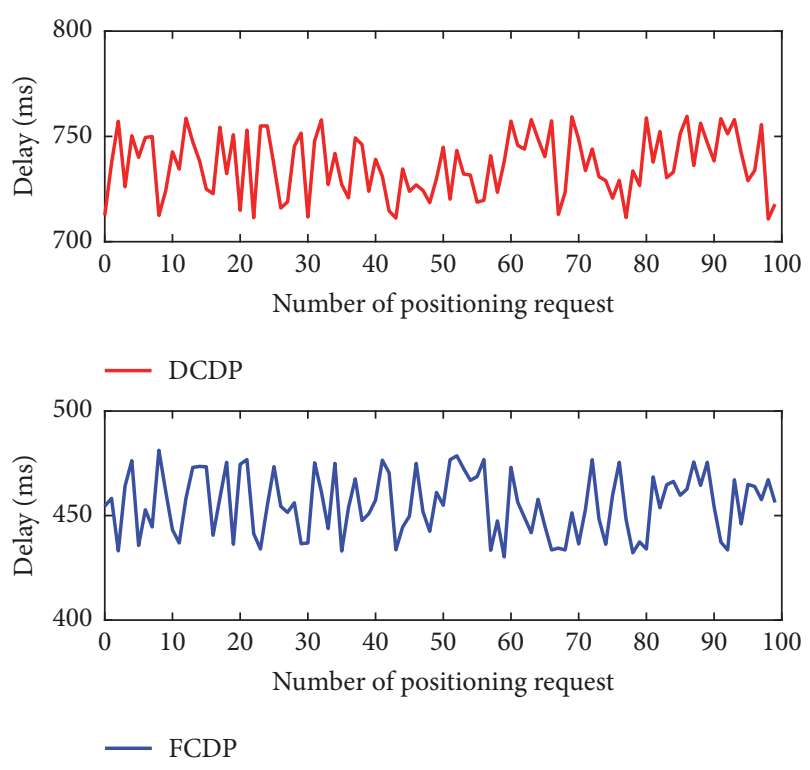

FIGURE 7: Delay of DCDP and FCDP methods.

\section{Data Availability}

No data were used to support this study.

\section{Conflicts of Interest}

The authors declare that they have no conflicts of interest.

\section{Acknowledgments}

This work is supported by the National Natural Science Foundation of China under Grant no. 61701020, the University of Science and Technology Beijing Project under Grant no. 04130017, the Fundamental Research Funds for the Central Universities under Grant no. FRF-BD-17-015A, and the Foundation of Beijing Engineering and Technology Center for Convergence Networks and Ubiquitous Services.

\section{References}

[1] M. Li, L. Qu, Q. Zhao, J. Guo, X. Su, and X. Li, "Precise point positioning with the BeiDou navigation satellite system," Sensors, vol. 14, no. 1, pp. 927-943, 2014.

[2] C. Han, Y. Yang, and Z. Cai, "BeiDou navigation satellite system and its time scales," Metrologia, vol. 48, no. 4, pp. S213-S218, 2011.

[3] W. Tang, C. Deng, C. Shi, and J. Liu, "Triple-frequency carrier ambiguity resolution for Beidou navigation satellite system," GPS Solutions, vol. 18, no. 3, pp. 335-344, 2014.

[4] Z. zhang, K. Hua, and S. Sun, "The Application of the Combination of BeiDou and GPS in the Civil Aviation," Automation and Instrumentation, p. S1, 2005.

[5] J. Wen, D. Wang, Y. Meng et al., "Application of beidou navigation satellite system to geological survey," Journal of Geomechanics, vol. 3, article 003, 2012.

[6] S. Baselga and L. García-Asenjo, "GNSS differential positioning by robust estimation," Journal of Surveying Engineering, vol. 134, no. 1, pp. 21-25, 2008.

[7] J. K. Choi, S. H. Park, D. J. Cho et al., "Correction error generation algorithm for differential positioning performance analysis of navigation equipment," in Proceedings of the International Conference on Control, Automation and Systems, ICCAS '08, pp. 1099-1103, IEEE, 2008.

[8] S. G. Jin, R. Jin, and D. Li, "Assessment of BeiDou differential code bias variations from multi-GNSS network observations," Annales Geophysicae, vol. 34, no. 2, pp. 259-269, 2016.

[9] C. Rizos, "Alternatives to current GPS-RTK services and some implications for CORS infrastructure and operations," GPS Solutions, vol. 11, no. 3, pp. 151-158, 2007.

[10] U. Vollath, A. Buecherl, H. Landau et al., "Multi-base RTK positioning using virtual reference stations," in Proceedings of the ION GPS, vol. 95, pp. 123-131, 2000.

[11] Y. C. Hu, M. Patel, D. Sabella et al., "Mobile edge computing-A key technology towards 5G," ETSI White Paper, vol. 11, no. 11, pp. 1-16, 2015.

[12] S. Floyd and V. Jacobson, "Random early detection gateways for congestion avoidance," IEEE/ACM Transactions on Networking, vol. 1, no. 4, pp. 397-413, 1993.

[13] P. Cheng, F. Ren, R. Shu et al., "Catch the Whole Lot in an Action: Rapid Precise Packet Loss Notification in Data Center," in Proceedings of the NSDI, pp. 71-28, 2014.

[14] A. Phanishayee, E. Krevat, V. Vasudevan et al., "Measurement and Analysis of TCP Throughput Collapse in Cluster-based Storage Systems," in Proceedings of the FAST, vol. 8, pp. 1-14, 2008.

[15] E. Krevat, V. Vasudevan, A. Phanishayee et al., "On applicationlevel approaches to avoiding TCP throughput collapse in cluster-based storage systems," in Proceedings of the 2nd International Petascale Data Storage Workshop, PDSW '07, held in Conjunction with Supercomputing '07, pp. 1-4, November 2007.

[16] F. Bonomi, R. Milito, P. Natarajan et al., "Fog computing: a platform for internet of things and analytics," in Big Data and Internet of Things: A Roadmap for Smart Environments, pp. 169186, Springer, Cham, Switzerland, 2014. 
[17] G. Hu, V. Khoo, P. Goh, and C. Law, "Performance of Singapore Integrated Multiple Reference Station Network (SIMRSN) for RTK Positioning," GPS Solutions, vol. 6, no. 1-2, pp. 65-71, 2002.

[18] P. V. W. Loomis, Carrier phase differential GPS corrections network: U.S. Patent 5,899,957 [P], 1999.

[19] B. Forssell, R. A. Harris, and M. Martin-Neira, "Carrier phase ambiguity resolution in GNSS-2," in Proceedings of the Proceedings of Ion Gps. Institute of Navigation, vol. 10, pp. 1727-1736, 1997.

[20] R. Odolinski, P. J. G. Teunissen, and D. Odijk, "Combined GPS+ BDS for short to long baseline RTK positioning," Measurement Science and Technology, vol. 26, no. 4, Article ID 045801, 2015.

[21] G. Lu, On-the-fly RTK positioning system with single frequency receiver: U.S. Patent 6,127,968, 2000.

[22] S. Zhao, Y. Chen, H. Zhang et al., "Differential GPS aided inertial navigation: a contemplative realtime approach," IFAC Proceedings Volumes, vol. 47, no. 3, pp. 8959-8964, 2014.

[23] W. Tang, L. Jin, and K. Xu, "Performance analysis of ionosphere monitoring with beidou CORS observational data," Journal of Navigation, vol. 67, no. 3, pp. 511-522, 2014.

[24] S. Yi, C. Li, and Q. Li, "A survey of fog computing: concepts, applications and issues," in Proceedings of the 2015 Workshop on Mobile Big Data, pp. 37-42, ACM, Hangzhou, China, June 2015.

[25] T. H. Luan, L. Gao, Z. Li et al., "Fog computing: Focusing on mobile users at the edge," 2015, https://arxiv.org/abs/1502.01815.

[26] S. Park and Y. Yoo, "Network intelligence based on network state information for connected vehicles utilizing fog computing," Mobile Information Systems, vol. 2017, Article ID 7479267, 9 pages, 2017.

[27] T. Rieckh, R. Anthes, W. Randel, S.-P. Ho, and U. Foelsche, "Tropospheric dry layers in the tropical western pacific: comparisons of gps radio occultation with multiple data sets," Atmospheric Measurement Techniques, vol. 10, no. 3, pp. 10931110, 2017.

[28] Y. P. Sun, Y. S. Zhang, E. S. Wang et al., "Design and positioning algorithm of BD-2/GPS combined system," Electronic Design Engineering, vol. 23, article 027, 2011. 


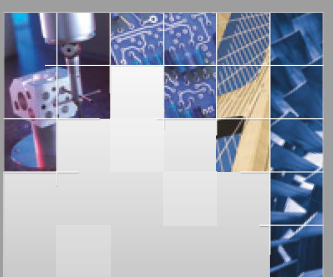

\section{Enfincering}
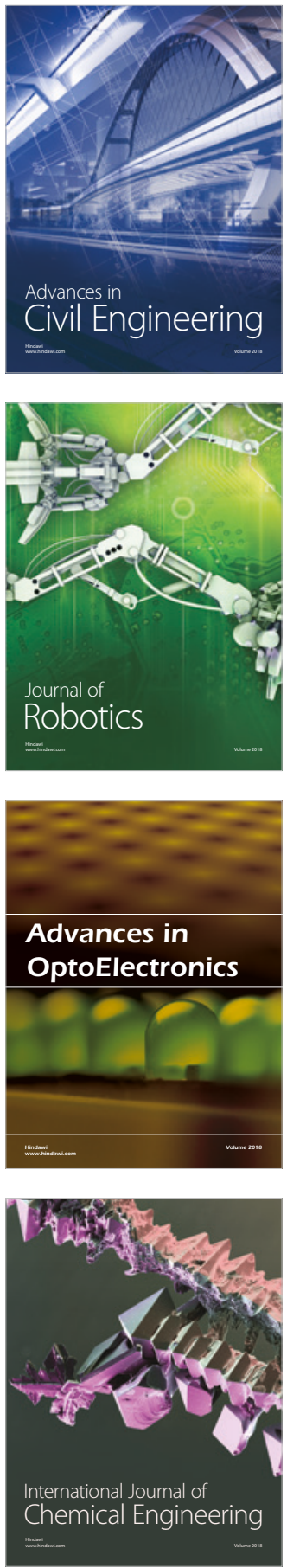

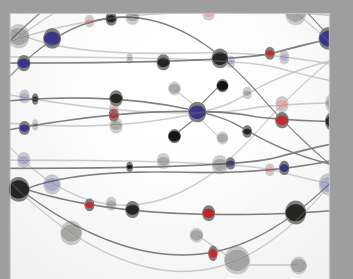

\section{Rotating \\ Machinery}

The Scientific World Journal

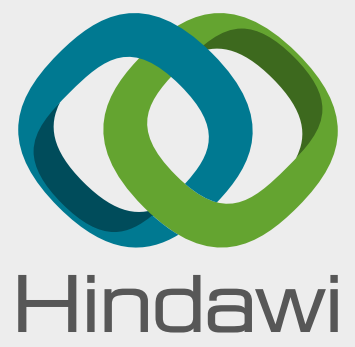

Submit your manuscripts at

www.hindawi.com
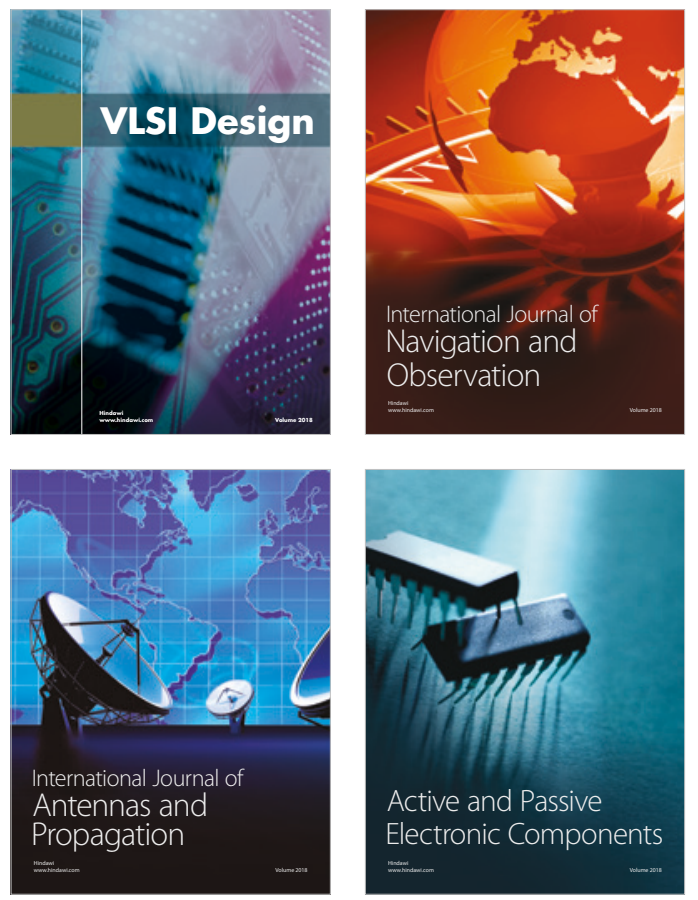
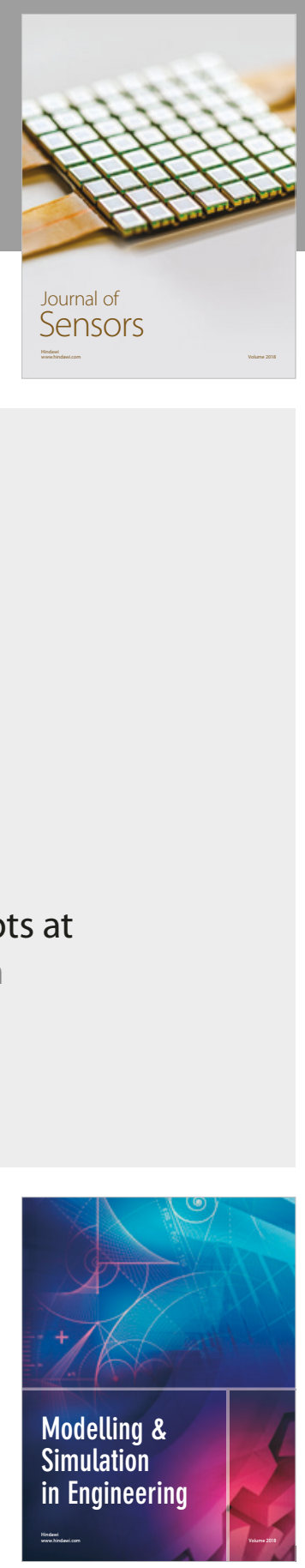

\section{Advances \\ Multimedia}
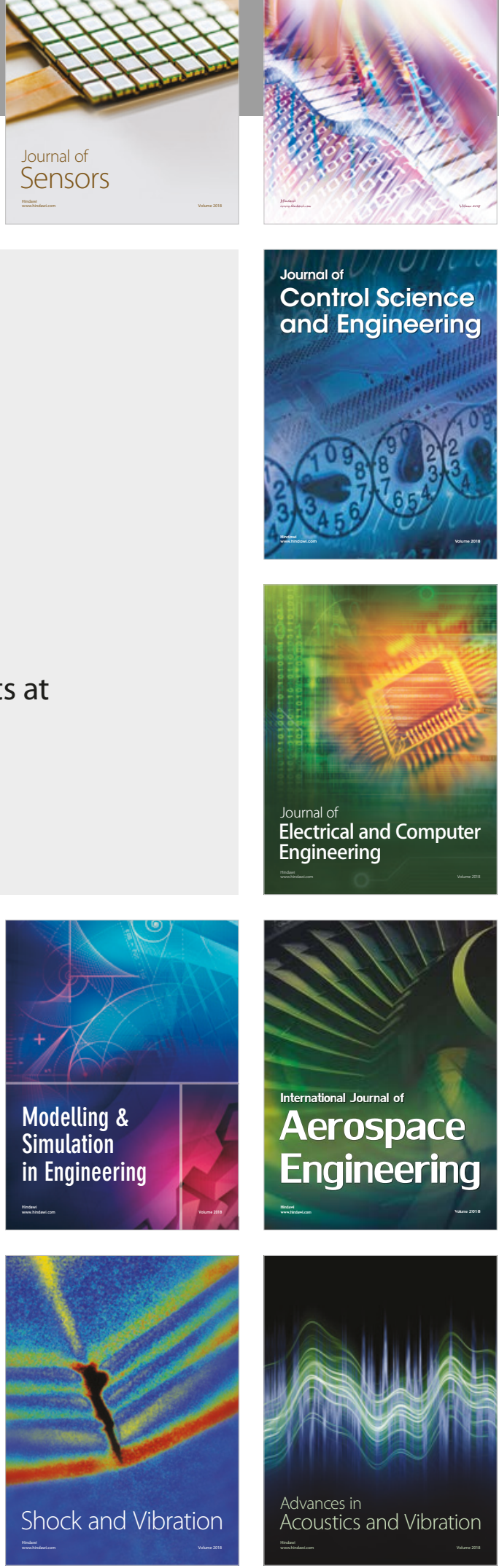